\title{
TTR
}

Traduction, terminologie, re?daction

\section{Kristal, Efraín. Invisible Work. Borges and Translation, Nashville, Vanderbilt University Press, xxii, 2002, 213 p.}

\section{Georges Bastin}

Volume 15, numéro 1, 1er semestre 2002

La traduction au Canada : Tendances et traditions

Translation in Canada : Trends and Traditions

URI : https://id.erudit.org/iderudit/006811ar

DOI : https://doi.org/10.7202/006811ar

Aller au sommaire du numéro

\section{Éditeur(s)}

Association canadienne de traductologie

ISSN

0835-8443 (imprimé)

1708-2188 (numérique)

Découvrir la revue

Citer ce compte rendu

Bastin, G. (2002). Compte rendu de [Kristal, Efraín. Invisible Work. Borges and Translation, Nashville, Vanderbilt University Press, xxii, 2002, 213 p.] TTR,

15(1), 247-251. https://doi.org/10.7202/006811ar d'utilisation que vous pouvez consulter en ligne.

https://apropos.erudit.org/fr/usagers/politique-dutilisation/ 
and I carry on a proud 4500-year-old tradition of linguisticallyenlightened intercultural communication. This book invites all its readers to belong to the same noble tradition.

\section{Ian Martin \\ English Department \\ Glendon College \\ York University}

\section{Kristal, Efraín. Invisible Work. Borges and Translation, Nashville, Vanderbilt University Press, xxii, 2002, 213 p.}

Outre le fait d'être des monuments de la littérature latino-américaine contemporains, quel autre trait partagent Gabriel García Márquez, Carlos Fuentes et Mario Vargas Llosa?

Celui de l'appropriation! García Márquez n'a-t-il pas transformé le comté de Yoknapatawpha de Faulkner en Macondo et fait de la ligne du temps de Buddenbrooks de Thomas Mann la colonne vertébrale généalogique de la famille Buendía? La mort d'Artemio Cruz de Fuentes n'est-elle pas une reprise du Citizen Cane d'Orson Welles? Vargas Llosa n'a-t-il pas réécrit Joseph Conrad et Victor Hugo entre autres?

C'est en tout cas ce qu'affirme Efraín Kristal pour expliquer l'héritage légué par Jorge Luis Borges (1899-1986) à la littérature latino-américaine et mondiale.

Professeur à l'UCLA et détenteur d'un doctorat de Stanford, Efraín Kristal est spécialiste de la littérature hispano-américaine des $\mathrm{XIX}^{\mathrm{e}}$ et $\mathrm{XX}^{\mathrm{e}}$ siècles et de littérature comparée. Il a notamment publié plusieurs ouvrages sur Mario Vargas Llosa dont Temptation of the Word (1998) et divers articles sur J.L. Borges.

Jorge Luis Borges, donc, le plus universel des écrivains latinoaméricains était copieur, adaptateur, faussaire, génie, éternel insatisfait, rêveur, misogyne, hétéro, narcissique ; telle apparaissait la personnalité du maître de la réécriture et de la retraduction.

« Je n'écris pas, je réécris. C'est ma mémoire qui produit mes phrases. J'ai tellement lu et tant entendu. Je l'avoue : je me répète. Je 
le confirme : je plagie. Nous sommes tous les héritiers de millions de scribes qui ont déjà écrit, longtemps avant nous, tout ce qui est essentiel. Nous sommes tous des copistes, et les histoires que nous inventons ont déjà été racontées. Il n'y a dorénavant plus d'idées originales. » (Jacques Chancel, Jorge Luis Borges Radioscopie, Paris, éditions du Rocher, 1999, pp. 74-76, cité p. 135).

À peine âgé de dix ans, Borges entreprend de traduire Oscar Wilde. Jusqu'à la fin de sa vie, alors qu'il préparait une version espagnole de Prose Edda, Borges a énormément traduit : des œuvres de Herman Melville, Ralph Waldo Emerson, Carlyle, Robert Louis Stevenson, Henri Michaux, Walt Whitman, Angelus Silesius et Snorri Sturluson; des nouvelles de Franz Kafka, Herman Melville, Jack London, Chesterton, Edgar Allan Poe, Villiers de l'Isle Adam, Guillaume Apollinaire, Nathaniel Hawthorne et Rudyard Kipling; et des poèmes de Wilhelm Klemm, André Gide, Wallace Stevens, Langston Hughes et Herman Hesse. Il a aussi traduit des essais et des chapitres de livres, ainsi que des centaines de passages d'ouvrages qu'il a cités dans ses anthologies et essais. Dans de nombreuses études, conférences et entrevues, Borges a analysé les versions des autres et s'est donné une vision de la traduction. Il prétendait que traduire pouvait améliorer l'original, que les versions contradictoires d'une même œuvre pouvaient être aussi valables et qu'un original pouvait être infidèle à sa traduction.

La version du Don Quixote de Pierre Ménard en particulier, ainsi que nombre de ses écrits et de ses traductions, sont qualifiés par Borges lui-même d' « œuvre invisible », de réalisation passée inaperçue, dissimulée et opaquée par une autre œuvre qu'elle a dépassée. D'où le titre de l'étude : Invisible Work. Borges and Translation.

L'objectif de l'ouvrage n'est pas de porter un jugement sur la valeur de la démarche traductionnelle de Borges ni sur sa pratique de traducteur, mais bien de les mettre au jour afin de démontrer leur impact sur la vision qu'avait Borges de la littérature comme art (pp. 135-136). Kristal cherche à prouver que la traduction, en tant que processus par lequel un écrivain remanie une séquence de mots en une autre, se trouve au cœur des réflexions de Borges sur l'écriture et de sa contribution à la littérature (xiii-xiv). Bref que les traductions de Borges, et sa vision de la traduction, font partie intégrante de son processus de création.

L'ouvrage se divise en trois chapitres : Borges et la traduction, 
Borges traducteur et La traduction dans le processus de création, où la traduction est aussi traitée en tant que sujet littéraire de la fiction de Borges puisque de nombreux personnages y sont des traducteurs et que de nombreuses histoires y sont présentées comme des pseudotraductions. Plusieurs des traductions de Borges, il ne s'agit en effet pas d'une étude exhaustive, y sont analysées à la lumière de la gestation et de l'édification de son monde littéraire. Les différences linguistiques y sont donc moins remarquées que « les élisions et les interpolations ».

Le premier chapitre rend compte des idées borgésiennes sur la traduction, de sa contribution originale à la théorie et à la critique littéraires. Pour ce faire, l'auteur ausculte Borges dans ses rapports avec le traduisible et l'intraduisible, et avec la traduction de ses propres œuvres. Il revisite le célèbre débat entre Arnold et Newman. Le sempiternel débat du littéral et du libre, de la forme et du contenu, du linguistique et du sens, revu et corrigé par deux professeurs britanniques à propos de la version anglaise de l'Iliade. Il écoute Borges parler des Mille et une nuits, applaudir Antoine Garland d'avoir, à l'instar des copistes du texte original du XIVe siècle, ajouté des contes et louer Richard Burton d'avoir inclus des interpolations personnelles. Borges lui-même recréera une traduction de Garland et inventera un conte qu'il attribuera à Burton! Kristal débouche ainsi sur la doctrine borgésienne de la traduction : "Borges préfère un traducteur qui falsifie (Madrus) à celui qui prétend capter la véracité de l'original », par contre, il rejette le plus fidèle (Littman) parce qu'il ne s'engage pas envers la littérature (p. 29). Borges n'a cependant jamais écrit de «traité » de la traduction. Il s'intéressait davantage à l'examen de cas particuliers qu'à des considérations d'ordre théorique. Il affectionnait la vision de Novalis. Le projet de Pierre Ménard s'inspire d'ailleurs de Novalis. Pour Borges, « la traduction n'est pas le transfert d'un texte d'une langue à une autre, mais la transformation d'un texte en un autre. [...] On peut traduire dans une même langue, mais on peut copier d'une langue à une autre. » (p. 32) Sans jamais offrir de méthode proprement dite, Borges utilisait le terme " préférence » pour qualifier les choix, les jugements et les discriminations d'un traducteur. Il optait donc pour une " méthode libre, ouverte voire enthousiaste aux transformations et aux modifications. » (p. 34)

Le second chapitre étudie les méthodes de travail de Borges traducteur au moyen de l'analyse d'un certain nombre de ses versions (poétiques et autres) narratives pour lesquelles il prit certaines libertés par rapport aux originaux. Fier que sa première publication eut été une 
traduction (The Happy Prince d'Oscar Wilde en 1910), Borges traduisait généralement seul, mais il a collaboré à de nombreux projets de traduction avec d'autres, dont sa mère Leonor Acevedo de Borges, son épouse María Kodama, son ami Adolfo Bioy Casares et le poète et éditeur Roberto Alifano. Les traductions placées sous la loupe de Kristal au long de ce chapitre sont nombreuses et variées. Tout d'abord la poésie de l'expressionnisme allemand et celle de Whitman. Ensuite «The Purloined Letter» de Poe, traduite selon la méthode de Baudelaire, soit l'amélioration du style incluant l'omission de détails, la contraction de passages et le changement de genre de personnages (vieille " manie » borgésienne déjà présente dans sa première traduction). Les traductions de Chesterton par Alfonso Reyes revues et corrigées par Borges et Bioy Casares, puis publiées comme étant les leurs... Des traductions de Kafka, dont des extraits de diverses œuvres posthumes réunis dans une nouvelle écrite par Borges, mais attribuée par ce dernier à Kafka. Et plusieurs autres. En conclusion, la méthode de travail de Borges repose sur un objectif principal : " créer une œuvre littéraire convaincante » (p. 87) et cinq stratégies : 1) l'élimination des redondances, 2) l'omission des " distractions textuelles " pour centrer l'attention du lecteur sur des aspects importants de l'avis de Borges, 3) l'ajout de nuances parfois très significatives, 4) la réécriture d'une œuvre à la lumière d'une autre, et 5) l'inclusion d'une traduction littérale dans l'une de ses propres œuvres. Soit une écriture créative.

Le dernier chapitre examine le rôle de la traduction dans certaines œuvres de fiction de Borges parmi les plus importantes. En premier lieu, " la majorité des nouvelles de Borges contiennent au moins un personnage traducteur » (p. 88). Ensuite, " nombre de ses histoires sont présentées comme des traductions, nombre de passages traduits sont insérés dans le corps de ses écrits, et nombre de traductions réelles ou imaginaires jouent un rôle dissimulé ou apparent dans la genèse ou le contenu de ses fictions » (p. 88). Par exemple, l'une des introductions les plus courantes des nouvelles de Borges est un texte bref par un narrateur qui introduit un manuscrit traduit, soit une pseudo traduction.

L'ouvrage se termine sur une postface intitulée « Borges et la philosophie » qui commente l'ouvrage de Barrenechea (1965). Selon cette dernière, "Borges est un écrivain admirable voué à détruire la réalité et à transformer l'Homme en une ombre » (cité p. 141). C'est un réaliste sceptique, un sceptique devenu ironique, qui affirme : "Le monde extérieur est comme nous le percevons ou l'imaginons. Il 
n'existe pas en dehors de nos esprits. La réalité et la fiction sont intimement liées. Nos idées sont des fictions créatives. » (cité p. 142) Un partisan de la contradiction aussi : « La contradiction est souvent une conviction intime. Je n'ai jamais cru ceux qui ont un point de vue univoque, ou ceux dont les affirmations sont toujours cohérentes. » (cité p. 142) Borges poussait très loin l'ironie : "J'affectionne vivre dans la perplexité, et je m'étonne que les gens me prennent au sérieux, j'ignore si je me prends moi-même au sérieux. » (cité p. 145)

Un personnage fascinant pour un ouvrage qui fascine. L'expert saura aussi apprécier le lourd appareil de notes (43 pages) et les très nombreuses citations de et sur Borges. Les autres découvriront un traducteur, pour paraphraser ce que dit Borges d'un de ses personnages, qui « n'a pas travaillé pour la postérité ni même pour Dieu, dont il ne connaissait rien des préférences littéraires. Minutieux, immobile, secret, il a tramé dans le temps son labyrinthe invisible. » (cité p. 133) Fausse modestie?

\section{Georges Bastin Université de Montréal}

\section{Loredana Polezzi. Translating Travel. Contemporary Italian Writing in English Translation, Aldershot, Ashgate "European Cultural transmission", 2001.}

Italy has always been an ongoing realm of fantasy, dreams and representations by foreign travelers. Both Italian literary critics and theorists have thus traditionally focused their attention on "foreign representations" of their "domestic culture". Yet Mrs. Loredana Polezzi, in a both thorough and original analysis, decided to look at the other side of the coin. The author hence studies travel writing in the Italian context from a radically new point of view, focusing on "domestic" - Italian - contemporary narratives about "foreign" cultures and on their English translations.

The following two questions raised respectively in the introduction and the conclusion, sum up the problematics of the book: 1) "Why does a genre, which in its international (and predominantly Anglo-Saxon) tradition is as popular in Italy as it is elsewhere in the world, fail to get recognition when it originates from 'home' writers?" (p.1); 2) On the other hand, "why should an Italian book on Tibet, or 